Special Distribution

Reporting Date: June 1975

Issued: June 1975

\title{
Potential Well Shapes for \\ Tensor Terms Involving Spin
}

by

P. W. Keaton, Jr.

This report was prepared as an account of work

sponsored by the United States Governsment. Neither

the United States nor the United States Energy

Research and Development Administration, nor any of

their employees, nor any of their contractors,

subcontractors, or their employees, makes any

warranty, express or implied or assumes any legt

Warranty, express implied, or assumes any legaf

lability or responsibility for the decuracy, completeness

or userulness of any information, apparatus, product or

process disclosed, or represents that its use would not

infringe privately owned rights.

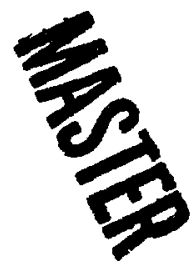


POTENTIAE WELL SHAPES FOR TENSOR TERMS INUOLVING SPIN

by

P. w. Keaton, Jr.

\section{ABSTRACT}

Potential well shapes for spin-orbit interactions are predicted using heuristic arguments. 'The results are generalized to interactions of the second rank in spin. Comparison with well known potential interactions are in agreement with the conclusions of this report.

\section{INTRODUCTION}

A suggestion by Ferani that the spin-orbit contribution to a nucleon-nucleus interaction is a surface effect ${ }^{l}$ can be generalized to deterrine the shizpe of a second-rank tensor term for any particlespin-nucleus interaction. This generalization reduces the number of ad hoc form factors that might be chosen for such a potential by establishing a consistent set of guidelines. For example, the same logic that leads to the Thomas form for the spinorbit term of a phenomenological potential describing deuteron-nucleus interactions also leads to a particular successive derivative form for the spinalignment tensor term. That is, the shape of the $\vec{S} \cdot \vec{L}$ term is connected to the shape of the central term $v_{c}$ by the relation $\left[\frac{1}{r} \frac{d v_{c}}{d r}\right]$, and the shape of the $\left\{(\hat{s} \cdot \hat{r})^{2}-\frac{1}{3} s(s+1)\right\}$ term is connected to the central term by the relation $\left[r \frac{d}{d r}\left(\frac{1}{r} \frac{d v_{c}}{d r}\right)\right]$, where $\vec{r}$ is the radius vector between the centers of the two interacting particles. This shape for the spinalignment tensor term agrees with the folding model for the deuteror-nucleus interaction ${ }^{2}$ and sith that for the classical electrostatic quadrupole interaction. Similarly, the same logic applied to spinspin interactions contributes a tensor term $\sim\left[r \frac{d}{d r}\left(\frac{1}{r} \frac{d V_{c}}{d r}\right)\right]\left\{\left(\vec{I}_{1} \cdot \hat{r}\right)\left(\vec{I}_{2} \cdot \hat{r}\right)-\frac{l}{3} \vec{I}_{1} \cdot \vec{I}_{2}\right\}$ to the potential. This shape for the spin-spin term agrees with the one pion exchange potential (OPEP) for nucleon-nucleon interactions and with that for classical magnetic dipole-dipole interactions.
Therefore, whenever a local potential containing nuclear spins is being devised without a specific theory, at least these simplicity arguments that will lead to form factors for the various spin contributions can be invoked. The purpose of this report is to generalize Fermi's suggestion by using simple algebraic techriques and to propose an internally consistent criterion for choosing well shapes that might be used to describe spin interactions.

\section{THE BASIC IDEA}

Around 1950 introduction of the nucleon-nucleus spin-orbit potential was necessary to explain the splitting of the energy levels in the nuclear shell mode ${ }^{3}$ and to explain nucleon polarization after elastically scattering from nuclei. ${ }^{4}$ Fermi concluded that an $\vec{S} \cdot \overrightarrow{\mathrm{L}}$ term would be predominantly a surface effect. ${ }^{1}$ He suggested that a nucleon passing through a uniform distribution of nucleons cannot locate the center of the distribution because the range of nucleon-nucleon forces is short (see Fig. 1). Therefore, any potential term containing angular momentum must vanish in the nuclear interior because the sign and magnitude of $\vec{L}$ cannot be determined. Near the surface, however, there is a preferred direction, namely, the gradient of the nucleon density $P(r)$. At the surface three vectors occur that might he used as "building blocks" to construct a potential term, which must be a scalar. These vectors are the particle spin $\vec{S}$, the particle momentum $\vec{p}$, and 

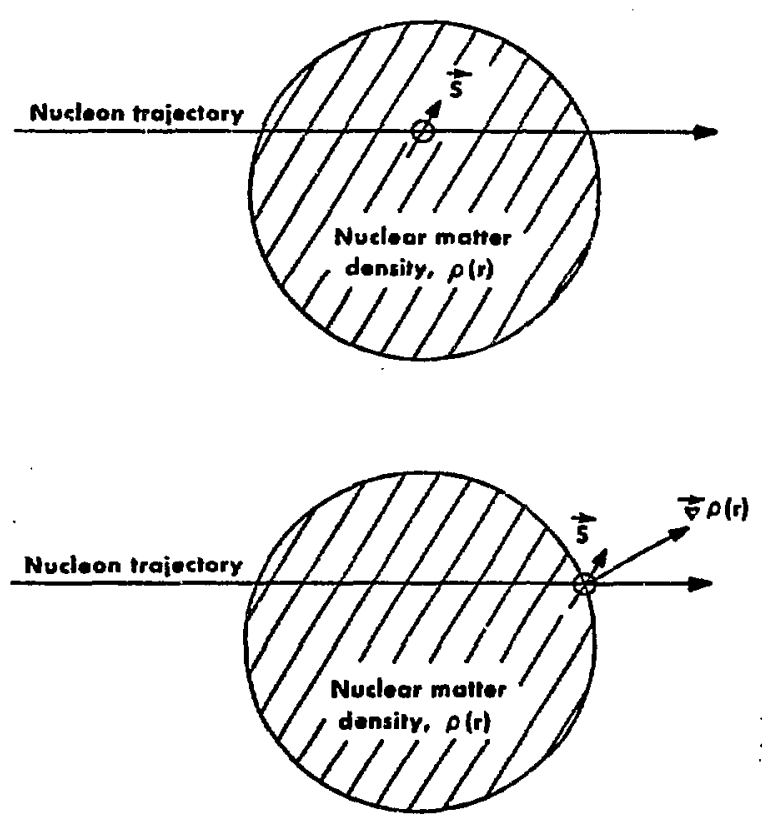

Fig. 1. A projectile of spin $\vec{s}$ immersed in a uniform nuclear density cannot locate the center of the nucleus and therefore cannot determine angular momentum $L=r \times \vec{p}$. However, at the surface the gradient of the nucleon density does supply a preferred direction.

the gradient of the nucleon density $\nabla \rho(r)$. The simplest combination is an inner product between $\vec{s}$ and $\vec{\nabla} \rho \times \vec{p}$, which, because $\vec{r} \times \vec{P}=\vec{L}$, results in

$$
\vec{s} \cdot \vec{\nabla} \rho \times \vec{p}=\frac{1}{r} \frac{d \rho}{d r} \vec{s} \cdot \vec{I}
$$

For short-range forces the shape of the nuclear potential $f(r)$ can be identified with the nucleon density distribution, and Eq. (1) yields the Thomas form for the spin-orbit term. Clearly all three available vectors were required to construct a scalar, and other simple combinations can be reduced to the form of Eq. (i).

The preceding argument is general in the sense that specifying the magnitude of $\vec{S}$ is not necessary and also in the sense that spin tensors of higher rank than one may be included. It therefore provides a guide for choosing the form factors of second-rank tensor terms and the only rationale for using a Thomas form for the $\overrightarrow{\mathrm{s}} \cdot \overrightarrow{\mathrm{L}}$ portion of a phenomenological deuteron optical potential.
To sumarize, when a particle of momentum $\vec{p}$ and spin $\vec{S}$ encounters a central potential $v_{c}=$ constant $x f(x)$, potential terms of rank 1,2 , etc. in spin can be constructed from $\vec{p}, \vec{s}$, and $\vec{\nabla} f(r)$. This construction consists of an invariant contraction of tensors that, in the case of rank 1 , is the scalar product of the two pseudovectors $\vec{s}$ and $\vec{\nabla} f(x) \times \vec{p}$. Each term of the potential is considered independently and is thexefore required to be even in time and parity.

INVARIANT CONTRACTIONS OF CARTESIAN TENSORS

The Cartesian elements $s_{i j}$ of a second-rank tensor can be constructed from vectors $\vec{A}$ and $\vec{B}$ in Cartesian space by

$$
S_{1 j}(\vec{A}, \vec{B})=\frac{1}{2}\left(A_{i} B_{j}+A_{j} B_{1}\right)-\frac{1}{3} \vec{A} \cdot \vec{B} \delta_{i j}
$$

where $\delta_{1 j}$ is the Kronecker delta and $A_{i}, B_{1}$ are components of the vectors $\vec{A}$ and $\vec{B}$, respectively. The invariant contraction of cartesian censors (or tensor scalar product) is designated by

$$
[\vec{A} \vec{B}]:\{\vec{C} \vec{D}\}=\sum_{i, j=x_{, y, z}} s_{i j}(\vec{A}, \vec{B}) S_{i j}(\vec{C}, \vec{D})
$$

and expanded to

$$
\begin{aligned}
{[\vec{A} \vec{B}]:[\vec{C} \vec{D}] } & =\left(A_{x} B_{x}-\frac{1}{3} \vec{A} \cdot \vec{B}\right)\left(C_{x} D_{x}-\frac{1}{3} \vec{C} \cdot \vec{D}\right) \\
& +\left(A_{y} B_{y}-\frac{1}{3} \vec{A} \cdot \vec{B}\right)\left(C_{y} D_{y}-\frac{1}{3} \vec{C} \cdot \vec{D}\right) \\
& +\left(A_{z} B_{z}-\frac{1}{3} \vec{A} \cdot \vec{B}\right)\left(C_{z} D_{z}-\frac{1}{3} \vec{C} \cdot \vec{D}\right) \\
& +\frac{1}{2}\left(A_{x} B_{y}+A_{y} B_{x}\right)\left(C_{x} D_{y}+C_{y} D_{x}\right) \\
& +\frac{1}{2}\left(A_{Y} B_{z}+A_{z} B_{y}\right)\left(C_{y} D_{z}+C_{z} D_{y}\right) \\
& +\frac{1}{2}\left(A_{z} B_{x}+A_{x} B_{z}\right)\left(C_{z} D_{x}+C_{x} D_{z}\right)
\end{aligned}
$$

By a simple matter of expansion, requiring only that vectors $\vec{B}$ and $\vec{C}$ collumute,

$$
\begin{aligned}
& (\vec{A} \cdot \vec{C})(\vec{B} \cdot \vec{D})=\frac{1}{3}(\vec{A} \cdot \vec{B})(\vec{C} \cdot \vec{D}) \\
& +\frac{1}{2}(\vec{A} \times \vec{B}) \cdot(\vec{C} \times \vec{D})+[\vec{A} \quad \vec{B}]:[\vec{C} \vec{D}] .
\end{aligned}
$$


similarly,

$$
\begin{aligned}
& (\vec{A} \cdot \vec{B})(\vec{C} \cdot \vec{D})+(\vec{A} \times \vec{B}) \cdot(\vec{C} \times \vec{D}) \\
& =(\vec{A} \cdot \vec{C})(\vec{B} \cdot \vec{D})+(\vec{A} \times \vec{C}) \cdot(\vec{B} \times \vec{D}),
\end{aligned}
$$

which is sometimes useful in manipulating expressions. Equation (5) can be used to express invariant contractions of tensors in terms of simple vector relations. The results are, of course, independent of the fact that cartesian tensors were used. The same results could have been obtained with spherical tensors by defining

$$
[\vec{A} \vec{B}]:[\vec{C} \vec{D}]=\sum_{q}(-1)^{q} T_{k q}(\vec{A}, \vec{B}) T_{k q}(\vec{C}, \vec{D})
$$

for $k=2$, as was done by Brink and Satchler. 5

For the deuteron-nuclear interaction, Satchler has shown ${ }^{6}$ that of the possible second-rank tensor terms only three need to be considered, namely,

$$
\begin{aligned}
& T_{r}=\left\{(\vec{s} \cdot \hat{r})^{2}-\frac{1}{3} s(s+1)\right\}, \\
& T_{p}=\left\{(\vec{S} \cdot \hat{p})^{2}-\frac{1}{3} s(s+1)\right\}, \text { and } \\
& T_{L}=\left\{(\vec{S} \cdot \vec{L})^{2}+\frac{1}{2} \vec{s} \cdot \vec{L}-\frac{1}{3} s(s+1) \ell(l+1)\right\} .
\end{aligned}
$$

These scalars are constructed from Eq. (5) with the four vectors $\vec{r}, \vec{p}, \vec{I}$, and $\vec{s}$ by forming $T_{r}=[\hat{r} \hat{r}]$ : [ $\vec{s} \vec{s}$ ], etc., where $\hat{r}=\vec{r} /|\vec{r}|$, etc. These results are consistent with this generalization as will be shown.

APPLICATION TO THE DEUTERON-NUCLEAR TENSOR POTENTIAI The three vectors $\vec{\nabla} f, \vec{s}$, and $\vec{p}$ are available to construct scalars for terms in the deuteronnuclear potential as described above. The simplest cases are selected for this report by requiring that each element of the second-rank tensors to be contracted be even in parity and time and contain an evan number of $\vec{s}$ vectors, and that each term contain at least one $f(x)$, which serves to connect the spin to the potential (thus ruling out, for example, $[\overrightarrow{\mathrm{p}} \overrightarrow{\mathrm{p}}]:[\overrightarrow{\mathrm{s}} \overrightarrow{\mathrm{s}}])$. In addition, the concept is illustrated by assuming spherical symmetry, $f(\vec{r})=$ f(r).
The results obtained by applying the above conditions on Eq. (5) are shown in Table'I. For example,

$$
\begin{aligned}
{\left[\vec{\nabla} f \vec{\nabla}_{f}\right]:[\vec{s} \vec{s}] } & =\left(\frac{1}{r} \frac{d f}{d r}\right)^{2}[\vec{z} \vec{r}]:[\vec{s} \vec{s}] \\
& =\left(\frac{1}{r} \frac{d f}{d r}\right)^{2}\left[(\vec{r} \cdot \vec{s})^{2}-\frac{1}{3} r^{2} s(s+1)\right] \\
& =\left(\frac{d f}{d r}\right)^{2} T_{r}
\end{aligned}
$$

where $T_{Y}$ is defined in Eq. (7). Also,

$$
\begin{aligned}
{[\vec{\nabla} \vec{\nabla} f]:[\vec{S} \vec{S}]=} & (\vec{\nabla} \cdot \vec{s})(\vec{\nabla} f \cdot \vec{s})-\frac{1}{3} s(s+1)\left(\nabla^{2} f\right) \\
= & \frac{1}{r} \frac{d}{d r}\left(\frac{1}{r} \frac{d f}{d r}\right)(\vec{s} \cdot \vec{r})^{2}+s(s+1)\left(\frac{1}{r} \frac{d f}{d r}\right) \\
& -\frac{1}{3} s(s+1) \nabla^{2} f \\
= & r \frac{d}{d r}\left(\frac{1}{r} \frac{d f}{d r}\right)_{r} .
\end{aligned}
$$

The invariant contractions of Table I always result in a form factor times $T_{r}, T_{p^{\prime}}$ or $T_{L^{\prime}}$ or a linear combination thereof as discussed in the previous section. Not shown in this table are contributions to the rank 1 and rank 0 terms derived from these calculations. The conclusion from this exercise is that possible form factors for $T_{r}$ are $\left(\frac{d f}{d r}\right)^{2}$, $\left(k \frac{d f}{d r}\right)^{2}$, and $r \frac{d}{d r}\left(\frac{1}{r} \frac{d f}{d r}\right)$, with no special preference among them. This problem has been studied using a folding model for the deuteron-nucleus interaction. ${ }^{2}$ Results show that the last term is in good agreement with the actual calculation and that there is negligible contribution to the potential from the $T_{p}$ and $T_{L}$ terms. This conclusion could not have been foreseen using the present approach.

Extracting the essence of the folding model by a Taylor expansion is worthwhile because it reinforces the choice of $r \frac{d}{d r}\left(\frac{1}{r} \frac{d v_{c}}{d r}\right)$ as a form factor and because the approach has more general applications. If $\vec{\alpha}$ is the radius vector between the neutron and proton, the effective potential between the deutezon and the nucleus is assumed to be

$$
v(\vec{r}, \vec{\alpha})=v_{p}\left(\vec{r}+\frac{1}{2} \vec{\alpha}\right)+v_{n}\left(\vec{r}-\frac{1}{2} \vec{\alpha}\right)
$$

where the subscripts $p$ and $n$ refer to the proton and neutron, respectively, and $v_{p}, v_{n}$ ar $z$ the 
TABLE I

DEUTYRON-NUCLEAR POTENTIAL FORM FACTORS

\begin{tabular}{|c|c|c|c|}
\hline Invariant Contractions & $T_{x}$ & $T_{p}$ & $T_{L}$ \\
\hline$[\vec{f} f \vec{f} f]:[\vec{s} \vec{s}]$ & $\left(\frac{d f}{d r}\right)^{2}$ & 0 & 0 \\
\hline$[\vec{\nabla} \vec{f} f\}:\{\vec{s} \vec{s}\}$ & $x \frac{d}{d x}\left(\frac{1}{x} \frac{d f}{d r}\right)$ & 0 & 0 \\
\hline$[\overrightarrow{f f} f \mathrm{f} f]:[\overrightarrow{\mathrm{p}} \times \overrightarrow{\mathrm{s}} \overrightarrow{\mathrm{p}} \times \overrightarrow{\mathrm{s}}]$ & 0 & $\left(k \frac{d f}{d x}\right)^{2}$ & $\left(\frac{1}{r} \frac{d f}{d r}\right)^{2}$ \\
\hline$[\vec{\nabla} \vec{\nabla} \mathbf{f}]:[\vec{p} \times \vec{s} \vec{p} \times \vec{s}]$ & 0 & $k^{2} x \frac{d}{d x}\left(\frac{1}{x} \frac{d f}{d x}\right)$ & $\frac{1}{r} \frac{d}{d r}\left(\frac{1}{r} \frac{d f}{d r}\right)$ \\
\hline$[\vec{\nabla} f \times \vec{p} \vec{b} f \times \vec{p}]=[\overrightarrow{\mathrm{s}} \overrightarrow{\mathrm{s}}]$ & 0 & 0 & $\left(\frac{1}{r} \frac{d f_{1}}{d r}\right)^{2}$ \\
\hline$[\vec{\nabla} f \times \vec{p} \vec{\nabla} f \times \vec{p}]:[\vec{p} \times \vec{s} \vec{p} \times \vec{s}]$ & 0 & $\ell(\ell+1)\left(\frac{k}{x} \frac{d f}{d r}\right)^{2}$ & $\left(\frac{k}{x} \frac{d f}{d x}\right)^{2}$ \\
\hline$[\vec{p} \vec{p}]:[\vec{s} \times \vec{f} f \vec{s} \times \vec{\nabla} f]$ & $\left(k \frac{d f}{d r}\right)^{2}$ & 0 & $\left(\frac{1}{x} \frac{d f}{d r}\right)^{2}$ \\
\hline
\end{tabular}

a Take $h=1$, so that $|\overrightarrow{\mathrm{p}}|=k$, and $\overrightarrow{\mathrm{L}} \times \overrightarrow{\mathrm{L}}=1 \overrightarrow{\mathrm{L}}$.

proton-, neutron-nuclear potentials. The nucleonnucleax potentials can be considered equal and comprised of a central part $v_{c}$, and a spin part $v_{s}(r) \vec{\sigma} \cdot \vec{L}$, where $\left|v_{S}(r) \vec{\sigma} \cdot \vec{L}\right|<<\left|v_{c}\right|$. with these assumptions, Eq. (10) becomes

$$
\begin{aligned}
v(\vec{r}, \vec{\alpha}) & \propto v_{c}\left(\left|\vec{r}+\frac{1}{2} \vec{\alpha}\right|\right)+v_{c}\left(\left|\vec{r}-\frac{1}{2} \vec{a}\right|\right) \\
& +\frac{1}{2} v_{s}(r)\left(\vec{\sigma}_{p}+\vec{\sigma}_{n}\right) \cdot \vec{L},
\end{aligned}
$$

where $\overrightarrow{\mathrm{L}}$ is the deuteron angular momentum and each nucleon contributes $\frac{1}{2} \vec{L}$ on the average. The central part of the equation can be expanded in is Taylor series:

$$
\begin{aligned}
& v_{c}\left(\left|\vec{r}+\frac{1}{2} \vec{\alpha}\right|\right)+v_{c}\left(\left|\vec{r}-\frac{1}{2} \vec{\alpha}\right|\right) \\
& \simeq\left\{2 v_{c}(r)+\frac{\alpha^{2}}{12}\left[\frac{1}{r^{2}} \frac{d}{d r}\left(r^{2} \frac{d v_{c}}{d r}\right)\right]\right\} \\
& +\frac{\alpha^{2}}{6}\left[r \frac{d}{d r}\left(\frac{1}{r} \frac{d v_{c}}{d r}\right)\right] P_{2}(\hat{r} * \hat{\alpha})
\end{aligned}
$$

The Legendre polynomial $P(\hat{r} \cdot \hat{\alpha})$ for $\ell=2$ has been used in Eq. (12). The interaction energy $w$ of the ceuteron can be considered classically as ${ }^{7}$

$$
w=\int|x(c)|^{2} v(\vec{x}, \vec{a}) d \vec{\alpha}
$$

where $x(\vec{\alpha})$ is the internal deuteron wave function and $|x(\vec{\alpha})|^{2}$ represents the nucleon density distribution. Taking ${ }^{B}$

$$
\begin{aligned}
& x(\vec{\alpha})=\frac{1}{\sqrt{4 \pi}}\left[\frac{u(\alpha)}{\alpha}+\frac{w(\alpha)}{\alpha \sqrt{\alpha}} s_{\mathrm{pn}}(\hat{\alpha})\right] \\
& s_{\mathrm{pn}}(\hat{\alpha})=\left\{3\left(\vec{\sigma}_{\mathrm{p}} \cdot \hat{\alpha}\right)\left(\vec{\sigma}_{\mathrm{n}} \cdot \hat{\alpha}\right)-\vec{\sigma}_{1} \cdot \vec{\sigma}_{2}\right\} \\
& =6\left\{(\vec{S} \cdot \hat{\alpha})^{2}-\frac{1}{3} \mathrm{~s}(\mathrm{~s}+1)\right\}
\end{aligned}
$$

where $\vec{s}=\frac{1}{2}\left(\vec{\sigma}_{p}+\vec{\sigma}_{n}\right)$, Eq. (13) can be calculated. Remember that $S_{p n}(\hat{\alpha}) \sim P_{2}(\hat{s} \cdot \hat{\alpha})$ if $\hat{s}$ is considered simpiy as an axis of sysunetry. Also note that $s_{p n}^{2}=8-2 s_{p n^{\prime}}$ and that ${ }^{8}$

$$
\frac{1}{2 \pi} \int_{0}^{2 \pi} s_{p n}(\hat{\alpha}) d \phi=s_{p n}(\hat{r}) P_{2}(\hat{r} \cdot \hat{\alpha}),
$$

where $\phi$ is the aximuthal angle of $\hat{\alpha}$ relative to a vector '́r.

Substituting Eqs. (11), (12), (14), and (15) into Eq. (13), 


$$
\begin{aligned}
W \simeq\left\{2 v_{c}(r)\right. & \left.+\frac{\left\langle\alpha^{2}>\right.}{12}\left[\frac{1}{r^{2}} \frac{d}{d r}\left(r^{2} \frac{d v_{c}}{d r}\right)\right]\right\} \\
& +v_{s}(R) \vec{s} \cdot \overrightarrow{\mathrm{L}} \\
& +\left[r \frac{d}{d r}\left(\frac{1}{r} \frac{d v_{c}}{d r}\right)\right]\left(\frac{1}{5 \sqrt{8}} \int_{0}^{\infty} u(\alpha) w(\alpha) \alpha^{2} d a\right) T_{r}
\end{aligned}
$$

where $\left\langle\alpha^{2}\right\rangle$ is the mean square radius of the deuteron. Thus $w$ is effectively the deuteron-nucleus interaction potential containing a central term in braces, a spin-orbit term, and $a\left[(\vec{s} \cdot \hat{r})^{2}-\frac{1}{3} s(s+1)\right]$ tern of the shape $\left[r \frac{\mathrm{d}}{\mathrm{dr}}\left(\frac{1}{\mathrm{r}} \frac{\mathrm{dv}}{\mathrm{dr}}\right)\right]$, which appears in Table I.

The form of Eq. (16) is correct for any density distribution containing a quadrupole term such as

$$
\rho(\vec{\alpha}) \sim a(\alpha)+b(\alpha) P_{2}(\hat{s} \cdot \hat{\alpha})
$$

which is located at position $\vec{r}$, where $|\vec{r}| \gg|\vec{\alpha}|$, and is located in a potential $v_{c}(r)$. In particular, an electric quadrupole distribution in a coulomb field caused by a point charge has form factors $v_{c} \sim 1 / r$ and $r \frac{d}{d r}\left(\frac{1}{r} \frac{d v_{c}}{d r}\right) \sim 1 / r^{3}$ as expected for $T_{r}$.

\section{APPLICATION TO THE OPEP}

The present generalization can be applied to the interaction of two spins, $\vec{I}_{1}$ and $\vec{I}_{2}$. As an example, consider

$$
\begin{aligned}
{\left[\vec{\nabla} \vec{V}_{f]:\left[\vec{I}_{1}\right.} \vec{I}_{2}\right]=} & \left(\vec{\nabla} \cdot \vec{I}_{1}\right)\left(\vec{\nabla} f \cdot \vec{I}_{2}\right)-\frac{1}{3}\left(\nabla^{2} f\right)\left(\vec{I}_{1} \cdot \vec{I}_{2}\right),(18) \\
= & x \frac{d}{d r}\left(\frac{1}{r} \frac{d f}{d r}\right)\left(\vec{I}_{1} \cdot \hat{r}\right)\left(\vec{I}_{2} \cdot \hat{r}\right) \\
& +\left(\frac{1}{r} \frac{d f}{d r}-\frac{1}{3} \nabla^{2} f\right) \vec{I}_{1} \cdot \vec{I}_{2} \\
= & x \frac{d}{d r}\left(\frac{1}{r} \frac{d f}{d r}\right)\left[\left(\vec{I}_{1} \cdot \hat{r}\right)\left(\vec{I}_{2} \cdot \hat{r}\right)-\frac{1}{3} \vec{I}_{1} \cdot \vec{I}_{2}\right] .
\end{aligned}
$$

where again Eq. (5) has been used. If the central force is derived from a yukawa potential,

$$
f(r) \sim \frac{e^{-l ! r}}{\mu r}
$$

then the spin-spin interaction should be of the forin

$$
r \frac{d}{d r}\left(\frac{1}{r} \frac{d f}{d r}\right)=\mu^{2} \frac{e^{-\mu r}}{\mu r}\left[1+\frac{3}{\mu r}+\frac{3}{(\mu r)^{2}}\right]
$$

which is correct for the OPEP of the nucleon-nucleon interaction. On the other hand, if the central force is simply of the $1 / x$ type, the spin-spin interaction is of the form $1 / x^{3}$, which is correct for magnetic dipole-dipole interactions.

The calculation procedure for the additional scalar terms for spin-spin interactions using $\vec{I}_{1}$. $\vec{I}_{2}$, $\vec{f} f_{r}$ and $\vec{p}$ is clear. However, a methodical appljcation of this approach has not yet been applied to the nucleon-nucleon problem.

CONCLUSION

Spin interactions have been treated as though they were dominated by regions of high potential gradients, and applications to several known problems have shown that this approach gives the correct form factors. Whenever a phenomenological potential containing nuclear spins is being devised, the simplicity arguments presented in this report can be used to suggest form factors for the various spin contributions.

\section{ACKNOWLEDGLENTS}

I wish to thank J. aammel, J. R. Nix, G. J. Stephenson, M. Bolsterli and B. F. Gibson for helpful discussions.

REFERENCES

1. E. Fermi, Nuovo Cimento Suppl. II, Serie 10, 18 (1955).

2. P. W. Keaton, Jr., and D. D. Armstrong, Phys. Rev. C 8, No. 5, 1692 (1973).

3. M. G. Mayer and J. H. D. Jensen, Elementary Theory of Nuclear Shell Structure (John wiley s Sons, Inc., New York, 1955).

4. E. Fermi, IL Nuovo cimento, $\underline{x}$, N. 4,407 (1954).

5. D. M. Brink and G. R. Satchler, Angular Momentum (Clarendon Press, Oxford, 1962) p. 124.

6. G. R. Satchler, Nucl. Phys. 21, 116 (1960).

7. J. D. Jackson, Classical Electradynamics (John wiley \& Suns, Inc., New York, 1962).

B. P. W. Keaton, Jr., E. Aufdembrink, and L. R. Veeser, "A Model for the Optical potential of Composite Particles" Los Alamos Scientific Iaboratory report LA-4379-kS (February 1970), PD. 36 and 60. 